OPEN ACCESS

Edited by:

David N. Kennedy,

University of Massachusetts Medical

School, USA

Reviewed by:

Hidetoshi lkeno,

University of Hyogo, Japan Frithjof Kruggel,

University of California, Irvine, USA

*Correspondence:

Ahmed Serag

a.f.serag@gmail.com

Received: 31 August 2016 Accepted: 05 January 2017 Published: 20 January 2017

Citation:

Serag A, Wilkinson AG, Telford EJ, Pataky R, Sparrow SA, Anblagan D,

Macnaught $G$, Semple SI and Boardman JP (2017) SEGMA: An Automatic SEGMentation Approach for Human Brain MRI Using Sliding Window and Random Forests.

Front. Neuroinform. 11:2. doi: 10.3389/fninf.2017.00002

\section{SEGMA: An Automatic SEGMentation Approach for Human Brain MRI Using Sliding Window and Random Forests}

\author{
Ahmed Serag ${ }^{1 *}$, Alastair G. Wilkinson ${ }^{2}$, Emma J. Telford ${ }^{1}$, Rozalia Pataky ${ }^{1}$, \\ Sarah A. Sparrow ${ }^{1}$, Devasuda Anblagan ${ }^{1,3}$, Gillian Macnaught ${ }^{4}$, Scott I. Semple S,5 $^{4,5}$ \\ James P. Boardman ${ }^{1,3}$ \\ ${ }^{1}$ MRC Centre for Reproductive Health, University of Edinburgh, Edinburgh, UK, ${ }^{2}$ Department of Radiology, Royal Hospital for \\ Sick Children, Edinburgh, UK, ${ }^{3}$ Centre for Clinical Brain Sciences, University of Edinburgh, Edinburgh, UK, ${ }^{4} \mathrm{Clinical}$ Research \\ Imaging Centre, University of Edinburgh, Edinburgh, UK, ${ }^{5}$ Centre for Cardiovascular Science, University of Edinburgh, \\ Edinburgh, UK
}

Quantitative volumes from brain magnetic resonance imaging (MRI) acquired across the life course may be useful for investigating long term effects of risk and resilience factors for brain development and healthy aging, and for understanding early life determinants of adult brain structure. Therefore, there is an increasing need for automated segmentation tools that can be applied to images acquired at different life stages. We developed an automatic segmentation method for human brain MRI, where a sliding window approach and a multi-class random forest classifier were applied to high-dimensional feature vectors for accurate segmentation. The method performed well on brain MRI data acquired from 179 individuals, analyzed in three age groups: newborns (38-42 weeks gestational age), children and adolescents (4-17 years) and adults (35-71 years). As the method can learn from partially labeled datasets, it can be used to segment large-scale datasets efficiently. It could also be applied to different populations and imaging modalities across the life course.

Keywords: brain, MRI, large-scale, life-course, sliding window, random forests, classification, tissue segmentation

\section{INTRODUCTION}

During early life, the brain undergoes significant morphological and functional changes, the integrity of which determines long-term neurological, cognitive and psychiatric functions (Tamnes et al., 2013). For instance, a wide range of problems including autism spectrum disorder, poor cognitive aging, stroke and neurodegenerative diseases of adulthood may have early life origins (McGurn et al., 2008; Shenkin et al., 2009; Hill et al., 2010; Wardlaw et al., 2011; Stoner et al., 2014). Improved understanding of cerebral structural changes across the life course may be useful for studying early life determinants and atypical trajectories that underlie these common problems.

Quantitative volumes from brain structural magnetic resonance imaging (MRI) acquired at different stages of life offer the possibility of new insight into cerebral phenotypes of disease, biomarkers for evaluating treatment protocols, and improved clinical decision-making and diagnosis. The literature presents a clear distinction between methods developed for different ages partly because the computational task is determined by properties of the acquired data and these 
are age-dependent (Cabezas et al., 2011; Despotovic et al., 2015; Išgum et al., 2015). For example, the infant brain presents challenges to automated segmentation algorithms developed for adult brain due to: wide variations in head size and shape in early life, rapid changes in tissue contrast associated with myelination, decreases in brain water, changes in tissue density, and relatively low contrast to noise ratio between gray matter (GM) and white matter (WM). Therefore, automated segmentation tools for modeling structure over years are limited, and this hampers research that would benefit from robust assessment of the newborn to the adult trajectory.

With regard to methodology, approaches for automatic segmentation of brain MRI can be classified into unsupervised (Cai et al., 2007; Leroy et al., 2011; Weglinski and Fabijanska, 2011; Gui et al., 2012) or supervised (Van Leemput et al., 2001; Fischl et al., 2002; Ashburner and Friston, 2005; Prastawa et al., 2005; Song et al., 2007; Altaye et al., 2008; Weisenfeld and Warfield, 2009; Shi et al., 2010; Kuklisova-Murgasova et al., 2011; Makropoulos et al., 2012; Serag et al., 2012b; Cardoso et al., 2013; Cherel et al., 2015; Moeskops et al., 2015; Wang et al., 2015; Loh et al., 2016) approaches. Supervised approaches have proven to be very successful in medical image segmentation (Aljabar et al., 2009; Lötjönen et al., 2010; Coupé et al., 2011; Rousseau et al., 2011; Kaba et al., 2014). However, as they rely on labeled training data (or atlases) to infer the labels of a test scan, most existing supervised approaches require a large number of training datasets to provide a reasonable level of accuracy and they usually carry a high computation cost due to their requirement of non-linear registrations between labeled data and the test scan (Iglesias and Sabuncu, 2015).

To address these challenges, here we describe a method for automatic brain segmentation of MR images, called SEGMA (SEGMentation Approach). SEGMA differs from current supervised approaches in the following ways. First, SEGMA uses a sparsity-based technique for training data selection by selecting training data samples that are "uniformly" distributed in the low-dimensional data space, and hence eliminates the need for target-specific training data (Serag et al., 2016). Second, SEGMA uses linear registration to provide an accurate segmentation (mainly to ensure the same orientation and size for all subjects). This is useful because it reduces computation time compared with most supervised methods which require nonlinear registrations between the training images and the target image. Finally, SEGMA uses a machine learning classification based on random forests (Breiman, 2001) where a class label for a given test voxel is determined based on its highdimensional feature representation. In addition to incorporating more information into the feature set (compared with methods that use voxel intensity information only), we use a sliding window technique that moves over all positions in the test image and classifies all voxels inside the window at once, instead of assigning labels on a voxel by voxel basis. This technique has the advantage of speeding-up the classification process while minimizing misclassifications compared with methods that use a global classifier (Iglesias et al., 2011; Vovk et al., 2011; Zikic et al., 2014). The feature extraction framework is illustrated in Figure 1.

\section{MATERIALS AND METHODS \\ Data And Image Acquisition}

The study includes brain imaging data from 179 subjects, spanning the ages of 0-71 years, from three MRI datasets.

\section{Dataset I}

The first dataset contained MR images from 66 infants: 56 preterms (mean post-menstrual age [PMA] at birth 29.23 weeks, range 23.28-34.84 weeks) were acquired at term equivalent age (mean PMA 39.84 weeks, range 38.00-42.71 weeks), and 10 healthy infants born at full term (>37 weeks' PMA). None of the infants had focal parenchymal cystic lesions. Participants of the newborns dataset were recruited to a larger study using MRI to study the effect of preterm birth on brain growth and longterm outcome. Ethical approval was granted by the National Research Ethics Service (South East Scotland Research Ethics Committee) and NHS Research and Development, and informed written parental consent was obtained.

A Siemens Magnetom Verio 3T MRI clinical scanner (Siemens Healthcare GmbH, Erlangen, Germany) and 12-channel phasedarray head coil were used to acquire: [1] T1-weighted (T1w) 3D MPRAGE: $\mathrm{TR}=1650 \mathrm{~ms}$, TE $=2.43 \mathrm{~ms}$, inversion time $=160$ $\mathrm{ms}$, flip angle $=9$ degrees, acquisition plane $=$ sagittal, voxel size $=1 \times 1 \times 1 \mathrm{~mm}^{3}, \mathrm{FOV}=256 \mathrm{~mm}$, acquired matrix $=256 \times 256$, acceleration factor (iPAT) $=2$; [2] T2-weighted (T2w) SPACE STIR: TR $=3800 \mathrm{~ms}, \mathrm{TE}=194 \mathrm{~ms}$, flip angle $=120$ degrees, acquisition plane $=$ sagittal, voxel size $=0.9 \times 0.9 \times 0.9 \mathrm{~mm}^{3}$, $\mathrm{FOV}=220 \mathrm{~mm}$, acquired matrix $=256 \times 218$. The image data used in this manuscript are available from the BRAINS repository (Job et al., 2017) (http://www.brainsimagebank.ac.uk).

Reference tissue segmentations for the dataset were generated using an Expectation-Maximization algorithm with tissue priors provided by the atlas from (Serag et al., 2012a,c). Ground truth accuracy of reference neonatal segmentations was evaluated by a radiologist experienced in neonatal brain MRI, who concluded that they were all plausible representations of anatomical classes. Quantitative evaluation of the reference segmentations was performed against manual segmentations from 9 subjects chosen at random. For each subject, three slices (those numbered as 25 th percentile, median and 75 th percentile of the slices containing brain tissue) were segmented. In order to remove bias toward any particular anatomical plane, three subjects were segmented in the axial plane, three in the coronal plane, and three in the sagittal plane. The quantitative analyses indicated high agreement for all tissues (mean Dice coefficient of $92 \%)$.

\section{Dataset II}

The second dataset contained T1w MRI scans and corresponding manual expert segmentation of 32 structures from 103 subjects (mean age 11.24 years, range $4.20-16.90$ years) publicly available from the Child and Adolescent NeuroDevelopment Initiative (CANDI) at University of Massachusetts Medical School (Frazier et al., 2008; Kennedy et al., 2012) (http://www.nitrc.org/projects/ candi_share). The data originates from four diagnostic groups: healthy controls $(N=29)$, schizophrenia $\operatorname{spectrum}(N=$ 


\begin{abstract}
Construct a feature vector for each voxel in a sliding window using intensity and gradient information
\end{abstract}

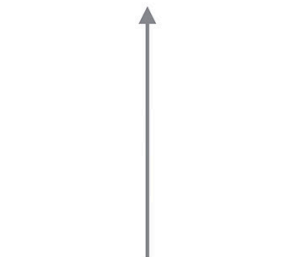

Slide a window over all positions

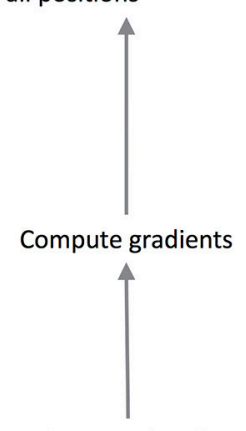

Brain extraction \& Bias field correction

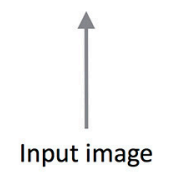

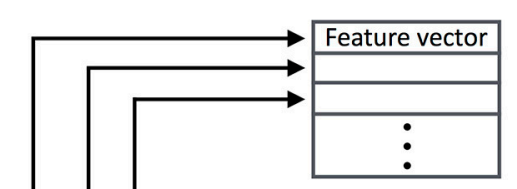

[ Feature vector, $f=$

intensity gradient

FIGURE 1 | Overview of the SEGMA feature extraction framework. The input test image is preprocessed for brain extraction and bias field correction, before computing gradients. Then, a sliding window is scanned across the input image at all positions where a feature vector for each voxel over the window is constructed using intensity and gradient information. The feature vectors are fed into a random forest classifier trained for structure / tissue classification.

20), Bipolar Disorder $(N=35)$, and Bipolar Disorder with psychosis $(N=19)$. The T1w images were acquired using a $1.5 \mathrm{~T}$ Signa scanner (GE Medical Systems, Milwaukee, USA) with the following parameters: a three-dimensional inversion recoveryprepared spoiled gradient recalled echo coronal series, number of slices $=124$, prep $=300 \mathrm{~ms}, \mathrm{TE}=1 \mathrm{~min}$, flip angle $=25$ degrees, $\mathrm{FOV}=240 \mathrm{~mm}^{2}$, slice thickness $=1.5 \mathrm{~mm}$, acquisition matrix $=$ $256 \times 192$, number of excitations $=2$.

\section{Dataset III}

The third dataset contained brain images and the corresponding manual expert segmentation of the whole brain into 32 structures from 18 healthy subjects including both adults and children; for the current study, we used only the adult data $(N=10$, mean age 38 , range $35-71$ years). The dataset is publicly available from the Internet Brain Segmentation Repository (www.nitrc. org/projects/ibsr) as IBSR v2.0 (Rohlfing, 2012). The T1w images were acquired using the following parameters: scanner/scan parameters unspecified, acquisition plane $=$ sagittal, number of slices $=128, \mathrm{FOV}=256 \times 256 \mathrm{~mm}$, voxel size $=0.8-1.0 \times$ $0.8-1.0 \times 1.5 \mathrm{~mm}^{3}$.

\section{Preprocessing}

For brain extraction, we used the brain masks which are provided with each dataset; except dataset I which was brain extracted using ALFA (Serag et al., 2016). All images from all datasets were corrected for intensity inhomogeneity using the N4 method (Tustison et al., 2010).

\section{Training Data}

The number of training examples often must be limited due to the costs associated with procuring, preparing and storing the training examples, and the computational costs associated with learning from them (Weiss and Provost, 2003). Therefore, we use in this work a sparsity-based technique to select a number of representative atlas images that capture population variability by determining a subset of $n$-dimensional samples that are "uniformly" distributed in the low-dimensional data space (Serag 
et al., 2016). The technique works by first linearly registering (12 degrees of freedom) all images from each dataset to an appropriate common coordinate space, and image intensities are normalized using the method described by (Nyul and Udupa, 2000). For dataset I, the 40 weeks PMA template from the $4 \mathrm{D}$ atlas (Serag et al., 2012a) was used as the common space, which is the closest age-matched template to the mean age of the cohort, while datasets II and III were aligned to the common space defined by the International Consortium for Brain Mapping (ICBM) atlas (Mazziotta et al., 2001). Then, all $N$ aligned images are considered as candidates for the subset of selected atlases. The closest image to the mean of the dataset is included as the first subset image. The consecutive images are selected sequentially, based on the distances to the images already assigned to the subset. Further details can be found in (Serag et al., 2016).

\section{Features}

We use machine learning to assign a label to all voxels in the test image, based on training a local classifier. Most existing methods for tissue classification only utilize information from voxel intensity, without considering other information. Here, in addition to voxel intensities, we incorporated various gradientbased features. Typically for each voxel $v$, a ten-dimensional feature vector $\mathbf{f}_{v}$ is extracted:

$$
\mathbf{f}_{v}=\left[\begin{array}{llllllllll}
I & I_{x} & I_{y} & I_{z} & r & \theta & \phi & I_{x x} & I_{y y} & I_{z z}
\end{array}\right]^{T}
$$

where $I$ is the gray scale intensity value, $I_{x}, I_{y}$ and $I_{z}$ are the norms of the first order derivatives, and $I_{x x}, I_{y y}$ and $I_{z z}$ are the norms of the second order derivatives. The image derivatives are calculated through the filters $\left[\begin{array}{lll}-1 & 0 & 1\end{array}\right]^{T}$ and $\left[\begin{array}{lll}-1 & 2 & -1\end{array}\right]^{T}$. The gradient magnitude $(r)$, azimuth angle $(\theta)$ and zenith angle $(\phi)$ are defined as follows:

$$
\begin{aligned}
r & =\sqrt{I_{x}^{2}+I_{y}^{2}+I_{z}^{2}} \\
\theta & =\tan ^{-1}\left(\frac{I_{y}}{I_{x}}\right) \\
\phi & =\cos ^{-1}\left(\frac{I_{z}}{r}\right)
\end{aligned}
$$

where $r \in[0, \infty), \theta \in[0,2 \pi)$, and $\phi \in[0, \pi]$.

\section{Random Forests}

In the last decade, random forests (RF) (Breiman, 2001) became a popular ensemble learning algorithm, as they achieve state-ofthe-art performance in numerous medical applications (Yi et al., 2009; Huang et al., 2010; Geremia et al., 2011; Mitra et al., 2014; Zikic et al., 2014; Tustison et al., 2015; Pereira et al., 2016). A RF ensemble classifier consists of multiple decision trees. In order to grow these ensembles, often random vectors are generated that govern the growth of each tree in the ensemble. Typically, each tree is trained by combining "bagging" (Breiman, 1996) (where a random selection is made from the examples in the training set) and random selection of a subset of features (Ho, 1998), which construct a collection of decision trees exhibiting controlled variation.
A test sample is pushed down to every decision tree of the random forest. When the sample ends up in one leaf node, the label of the training sample of that node it is assigned to the test sample as tree decision. Then, the final predicted class for a test sample is obtained by combining, in a voting procedure, the predictions of all individual trees. More details on decision forests for computer vision and medical image analysis can be found in Criminisi and Shotton (2013).

\section{Sliding-Window Based Classification}

A sliding window is used to move over all possible positions in the test image, and for each window, the voxels inside the window are classified into different tissues or structures. The vector in equation (1) represents the test sample for one voxel in a window, where the number of test samples is equal to the window size $w$. The training samples come from the voxels of the aligned atlas images that are located at the same location as the voxels belonging to the test window. This means that the number of training samples per window is equal to $k \times w$, where $k$ is the number of training atlases and $w$ is the window size, e.g., $5 \times 5 \times 5$, or $7 \times 7 \times 7$, etc.

A local RF classifier is then used to assign each voxel in the test image to a segmentation class. Figure 2 shows an example of classifying one test window. The SEGMA algorithm is summarized in Algorithm 1.

\section{Algorithm 1. SEGMA algorithm}

Set $\mathbf{f}_{v}$ to represent a feature vector for a voxel $v$ Set $c_{v}$ to represent a segmentation class for a voxel $v$ Set $k$ to represent the number of training data Set $w$ to represent the sliding window size for each window $W$ do

Construct the training data matrix $\mathcal{T}_{W}^{\text {Train }}=\left\{\mathbf{f}_{v}^{j} \mid j=\right.$ $1, \ldots, k ; v=1, \ldots, w\}$

Train the $R F_{W}$ classifier for window $W$ using $\mathcal{T}_{W}^{\text {Train }}$ Construct the test data matrix $\mathcal{T}_{W}^{\text {Test }}=\left\{\mathbf{f}_{v} \mid v=\right.$ $1, \ldots, w\}$

Determine the labels $c_{v}$ for all voxels inside the test window $W$ by applying $R F_{W}$ to $\mathcal{T}_{W}^{\text {Test }}$

end

\section{Evaluation}

A leave-one-out cross-validation procedure was performed for every dataset. Each subject from a dataset in turn was left out as a test sample and the remaining subjects were used as the training data where a subset of $k$ atlases is selected. The comparison between automatic $(A)$ and reference $(M)$ segmentations was performed using the Dice coefficient $(D C)$ (Dice, 1945) which measures the extent of spatial overlap between two binary images, with range 0 (no overlap) to 1 (perfect agreement). The Dice values are expressed as a percentage and obtained using the following equation:

$$
D C(A, M)=\frac{2|A \cap M|}{|A|+|M|} \times 100
$$




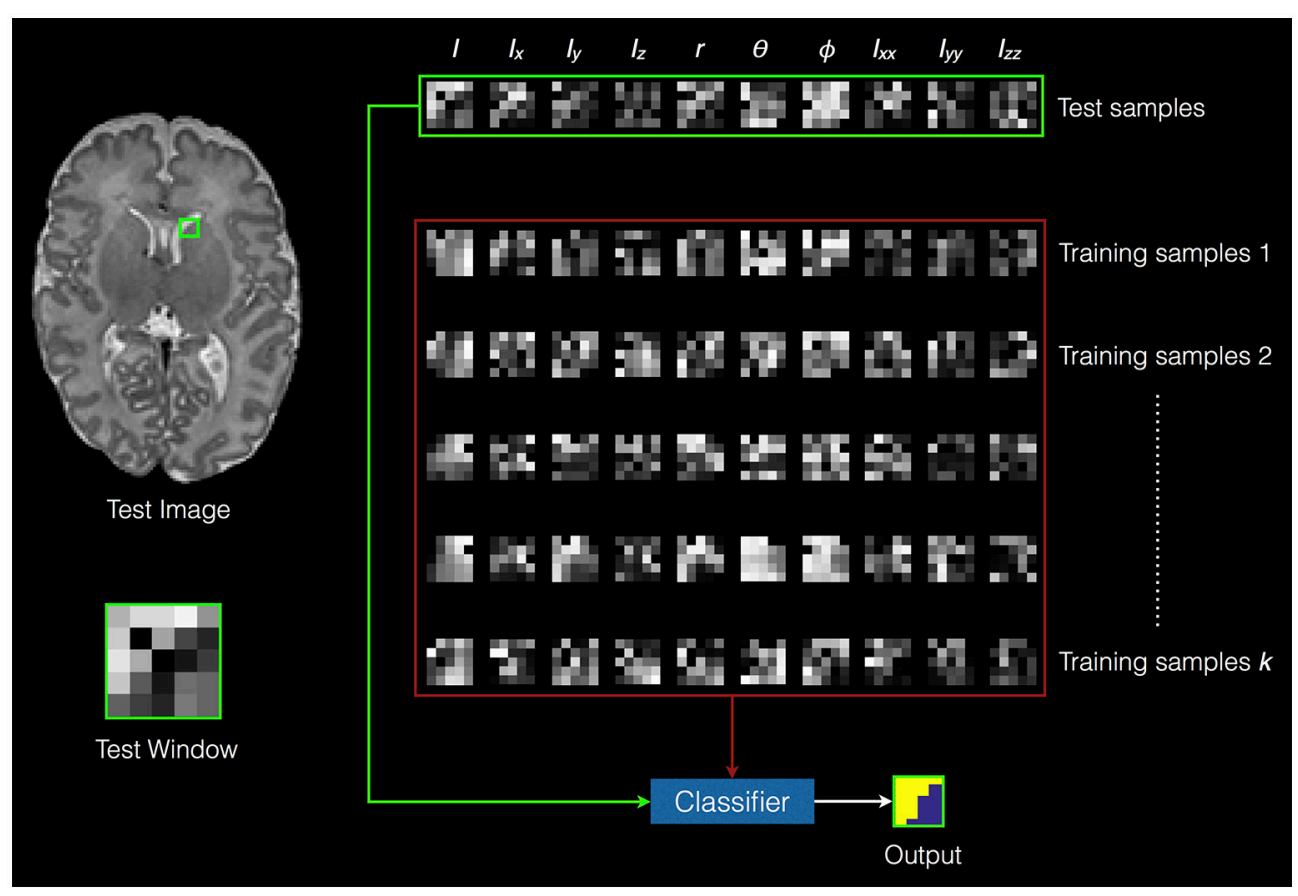

FIGURE 2 | An example of classifying one test window. The green square in the test image represents the test window. The green rectangle represents the extracted features from the test window (i.e., test samples). The red rectangle represents the extracted features from training data (i.e., training samples). The voxels inside the test window are classified into different classes based on training the random forest classifier using the training samples.

\section{Comparison against Other Methods}

We compared SEGMA against commonly used segmentation methods: Majority Vote (MV) (Rohlfing et al., 2004; Heckemann et al., 2006), Simultaneous Truth And Performance Level Estimation (STAPLE) (Warfield et al., 2004). The registration scheme for these methods is based on non-linear image deformation (Rueckert et al., 1999; Modat et al., 2010).

To compare SEGMA against other RF segmentation methods, we implemented a global RF classifier, similar to (Iglesias et al., 2011; Zikic et al., 2014), and experimented training it using intensity and gradient-based features, and intensity feature only. Non-linear registration was used as above to map the training images to the test image coordinate space, and the RF classifier was trained using 100,000 randomly sampled voxels from each training image.

\section{Statistical Analyses}

To test for differences between segmentation results, $t$-tests were used for normally distributed data, and Mann Whitney U was used to compare non-normal distributions (Shapiro-Wilk normality test was used). $P<0.05$ were considered significant after controlling for Type I error using false discovery rate (FDR).

\section{RESULTS}

To evaluate segmentation performance across the life course, SEGMA was applied to three publicly available datasets that provide MR brain images at different stages of the life course: neonatal period (38-42 weeks gestational age), childhood and adolescence (4-17 years), and adulthood (35-71 years). Figure 3 shows examples of brain segmentation results across the life course, and Figure 4 shows the resulting Dice coefficient (i.e., the agreement between the automatic and reference segmentations).

\section{Brain Segmentation in Neonatal Period}

We first applied the proposed segmentation method to a neonatal cohort (dataset I) consisting of $66 \mathrm{MR}$ images and associated segmentation of the following tissues / structures: brainstem, cerebellum, cortex or GM, cerebrospinal fluid (CSF), deep GM and WM. Quantitative analyses (Figure 4) indicated high accuracy for all tissues and structures with a mean Dice coefficient of $91 \%$.

The highest accuracies obtained for brainstem, cerebellum, deep GM, and WM with mean Dice coefficient of 90-94\%, while cortex and CSF had average Dice coefficients of 89 and $85 \%$, respectively.

\section{Brain Segmentation in Childhood and Adolescence}

To examine the performance of SEGMA in childhood and adolescence, we used 103 MR images from subjects aged 417 years (dataset II) with associated anatomical segmentation of 32 structures. Quantitative analyses (Figure 4) indicated high accuracy for all tissues and structures with a mean Dice coefficient of $86 \%$. Nine structures had an average Dice coefficient higher than 90\%, 7 structures had an average Dice coefficient 

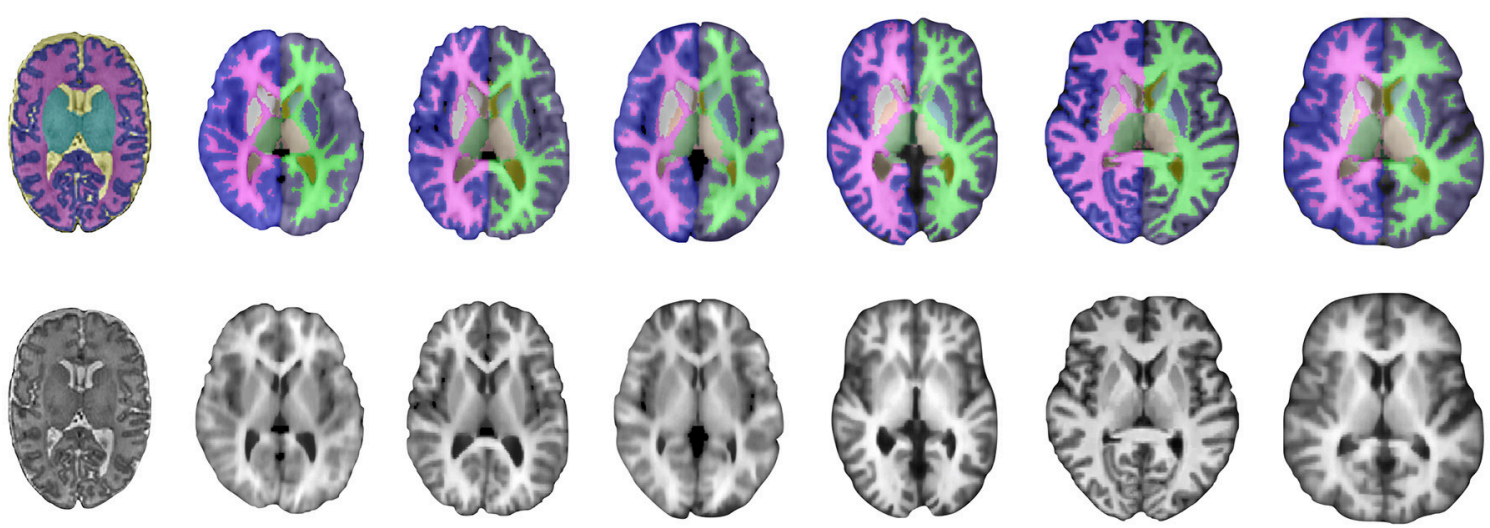

0 years

4 years

10 years

15 years

35 years

59 years

71 years

Adulthood

FIGURE 3 | Examples of brain segmentation results across the life course (axial view) using SEGMA. The automated segmentation is based on T2-weighted scans for the neonatal period and T1-weighted scans for the rest of growth stages. The images are taken from single subjects at the shown ages, where neonatal period images come from dataset I; childhood and adolescence images come from dataset II; and adulthood images come from dataset III.
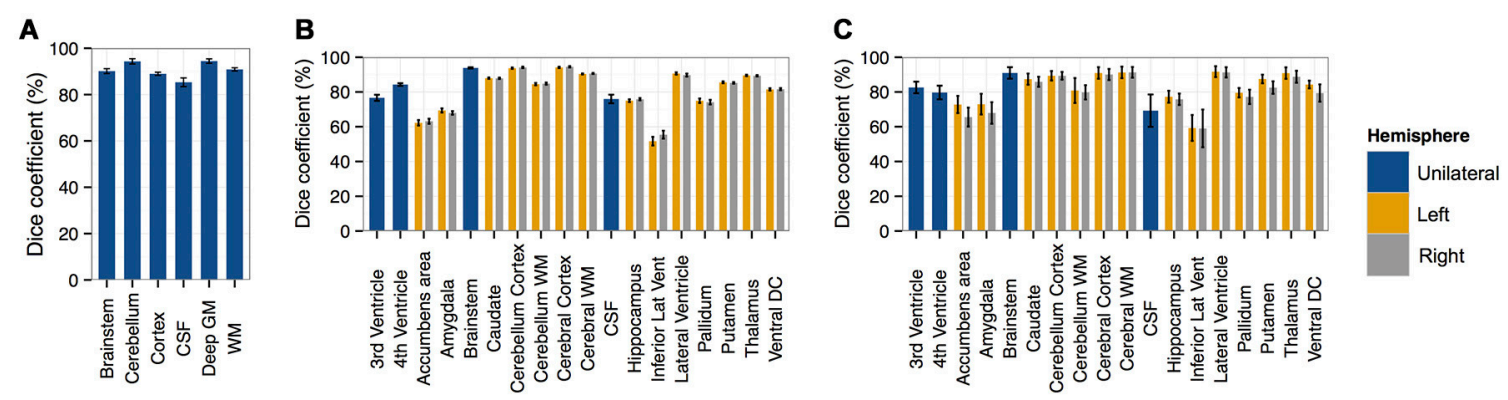

FIGURE 4 | Bar plots of the Dice coefficient (with standard deviation as error bar) comparing segmentations derived from SEGMA with reference segmentations using (A) dataset I [neonatal period], (B) dataset II [childhood and adolescence], and (C) dataset III [adulthood].

of $79-89 \%$, and 2 structures had an average Dice coefficient of $51-67 \%$.

\section{Brains Segmentation in Adulthood}

A dataset (dataset III) consisting of MR images and corresponding anatomical segmentation of 32 structures from 10 subjects (aged 38-71 years) was used to examine the performance of the segmentation algorithm in adulthood. Quantitative analyses (Figure 4) indicated high accuracy of $83 \%$. Seven structures had an average Dice coefficient higher than $90 \%, 9$ structures had an average Dice coefficient of $75-89 \%$, and 2 structures had an average Dice coefficient of $49-57 \%$.

\section{Comparison against Other Methods}

SEGMA was compared with two commonly used segmentation methods [Majority Vote (MV) (Rohlfing et al., 2004; Heckemann et al., 2006), Simultaneous Truth And Performance Level Estimation (STAPLE) (Warfield et al., 2004)], and other RF-based segmentation methods. SEGMA improved overall segmentation accuracy compared with MV, STAPLE, global-RF-1 (trained using intensity and gradient features), and global- RF- 2 (trained using intensity feature only); Table 1 shows Dice coefficients averaged over all structures, generated by each segmentation method and applied to datasets I, II and III. $(P<0.001$; after FDR correction).

\section{Reproducibility}

As dataset I (neonatal period) included T1-weighted (T1w) and T2-weighetd (T2w) MR imaging, we used it to test the reproducibility of SEGMA across different MR modalities by segmenting the newborn brain using information from T1w and T2w data separately (Figure 5). SEGMA provided consistent 
TABLE 1 | Dice coefficients averaged over all structures for datasets I, II, and III.

\begin{tabular}{lccccc}
\hline Dataset & SEGMA \% & $\begin{array}{l}\text { Global- } \\
\text { RF-1 \% }\end{array}$ & $\begin{array}{l}\text { Global- } \\
\text { RF-2 \% }\end{array}$ & MV \% & STAPLE \% \\
\hline I & $\mathbf{9 0 . 6 8}$ & 85.29 & 84.22 & 86.97 & 87.01 \\
II & $\mathbf{8 6 . 0 5}$ & 78.98 & 74.90 & 81.75 & 79.17 \\
III & $\mathbf{8 2 . 5 6}$ & 78.75 & 76.02 & 77.13 & 77.54 \\
\hline
\end{tabular}

SEGMA is compared with MV, STAPLE, global-RF-1, and global-RF-2.

segmentation results across different structural MRI modalities of the newborn brain. There was no statistically significant difference between mean Dice scores estimated from the two groups $(P=0.8977)$.

\section{Influence of Parameters}

We evaluated the influence of size of training data on segmentation accuracy, and found that increasing the size of the training data improves segmentation accuracy, evidenced by the increase in average Dice coefficient from $88 \%$ (7\% training data) to $91 \%$ ( $30 \%$ training data) for neonates, and from $83 \%$ (5\% training data) to $86 \%$ (20\% training data) for children and adolescents. From our experiments, 5-10 training images were sufficient to yield accurate results.

Forest parameters such as tree depth and number of samples per leaf node were set according to pervious work (Geremia et al., 2011; Zikic et al., 2014; Wang et al., 2015), and in this work, we only evaluated the influence of number of trees on segmentation accuracy. The number of trees in the forest characterizes the generalization power. As the number of trees becomes large, segmentation accuracy increases, but training time increases and a threshold value is reached after which further improvement is not achieved. In this work, number of trees was set to 10 .

With regard to window size, the smaller the window, the longer the classification time. Hence, window size needs to be chosen carefully as it provides a balance between accuracy and speed. Therefore, in this paper, we select the window size as $5 \times 5 \times 5$.

\section{Relative Importance of Features}

As partial volume effects in neonatal brain MRI present challenges for automatic segmentation methods, we evaluated the influence of each of the features on segmentation accuracy of the neonatal brain (dataset I). This was done by dropping one or a group of the ten features and running segmentation with the remaining features (features of the same type were dropped together). Therefore, an approximation of relative importance of each feature was obtained. Our experiments show that dropping the intensity feature significantly hinders the segmentation accuracy (Figure 6A), whilst the accuracy is improved by incorporating gradient-based features. When all of the features are used, SEGMA yielded higher accuracy than each individual category $(P<0.001$; after FDR correction). Figure 6B also shows an example of the automatic neonatal cortical GM segmentation and how the dropping of each of the ten features affects the segmentation accuracy.
We then analyzed the edge detection for various regions based on using all features (intensity combined with gradients) and gray scale intensity only. Figure 7 shows that gradient-based features improved edge detection for various regions of the adult and neonatal brain.

\section{Computation Time}

One classification task on a 64-bit iMac ${ }^{\circledR}\left(\right.$ Intel ${ }^{\circledR}$ Core i7 @ $3.5 \mathrm{GHz} \times 4.32 \mathrm{~GB}$ RAM) takes 5-7 min. The classification has benefited much from the sliding window strategy used. This is because instead of performing the classification in a voxel-wise manner, this is done for a batch of voxels at once. Assuming a window size of $5 \times 5 \times 5$, the classification time is decreased by 125 -folds. In addition, multi-core processing or computer clusters could greatly enhance the speed; and then one brain classification could be performed in about (or less than) $1 \mathrm{~min}$.

\section{DISCUSSION}

In this article, we present a new method for MRI brain segmentation (SEGMentation Approach, SEGMA). SEGMA was evaluated on three different datasets (span the ages 0-71 years) that provide different challenges to the brain segmentation task, and accurate results were obtained at all stages of development.

The method is trained using partially labeled datasets where a relatively small number of manually labeled images from the population under study are sufficient to provide accurate results. It is possible that training the method with a larger dataset might increase the segmentation accuracy. However, our goal was to design a methodology that can provide an acceptable, yet high accuracy result using a small number of training images (and thence a low computation cost).

The relatively lower performance for CSF could be caused by its bordering with GM (which is a complex shape). The boundary between GM and CSF is especially difficult to identify inside the sulci, where it is often poorly visible. In addition, the relatively lower performance for the children and adolescence, and adult datasets compared with the neonatal dataset could be attributable to scanner strength. Yet, the results obtained are comparable with those obtained using other methods tested on the same datasets (Rousseau et al., 2011; Zikic et al., 2014).

SEGMA uses a local RF classifier (trained by information from neighboring voxels in the same window) to assign a label to each voxel, which makes it less susceptible to classification errors such as the partial volume misclassification on the CSF-GM and CSF-background boundaries (Kuklisova-Murgasova et al., 2011; Cardoso et al., 2013; Išgum et al., 2015; Moeskops et al., 2015). We chose to use random forests as the classification technique since they naturally handle multi-class classification problems and are accurate and fast (Huang et al., 2010; Geremia et al., 2011; Criminisi and Shotton, 2013). Also, the sliding window plays an important role in significantly speeding up the classification task (compared to voxel-wise approaches).

The method provides an accurate segmentation using only linear registration, which ensures the same orientation and size for all subjects. This is an advantage compared with most supervised methods, which require non-linear registrations 


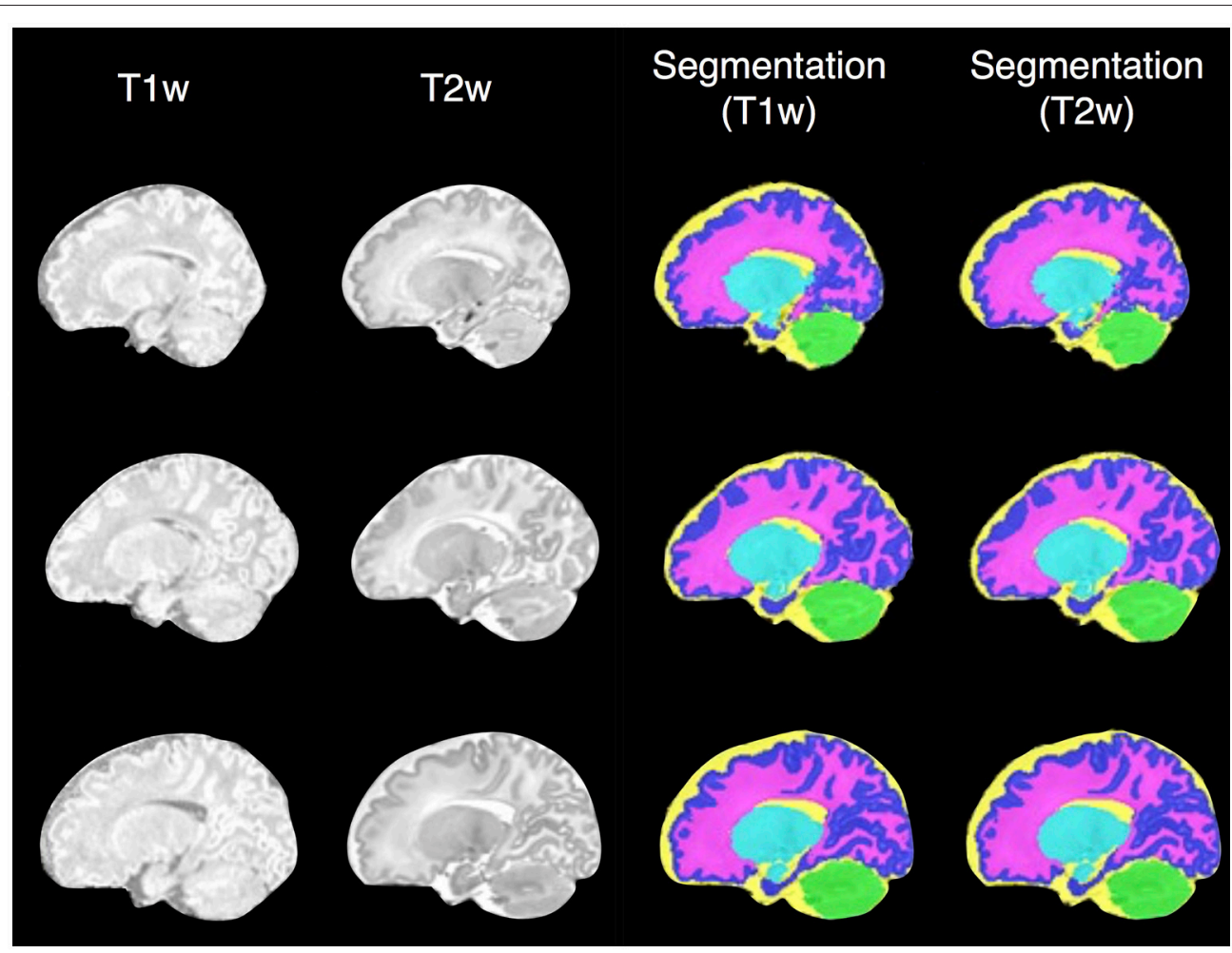

FIGURE 5 | Examples of SEGMA's output segmentation results (sagittal view) using T1-weighted (T1w) and T2-weighted (T2w) MR individually.

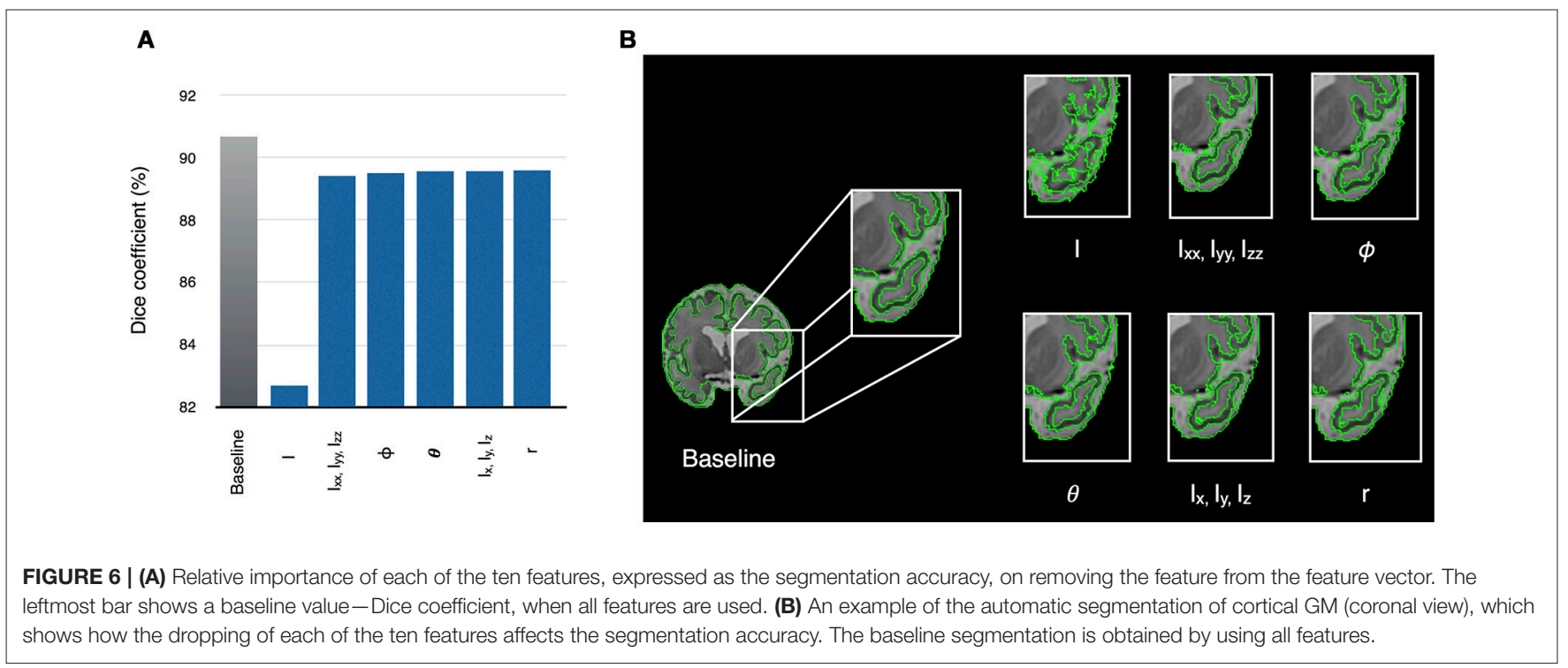

between the training images and the test image which increases segmentation time to several hours thereby compromising clinical utility (Iglesias and Sabuncu, 2015). SEGMA also has the advantage of providing an accurate segmentation using a single modality (which is important as the available data might be limited to one modality), and features that characterize object appearance and shape (intensity and gradients). However, the method is flexible and new features can easily be added to the high-dimensional feature vector.
To conclude, we present a method for segmentation of human brain MRI that is robust and provides accurate and consistent results across different age groups and modalities. As SEGMA can learn from partially labeled datasets, it can be used to segment large-scale datasets efficiently. The idea of SEGMA is generic and could be applied to different populations and imaging modalities across the life course. SEGMA is available to the research community at http://brainsquare. org. 


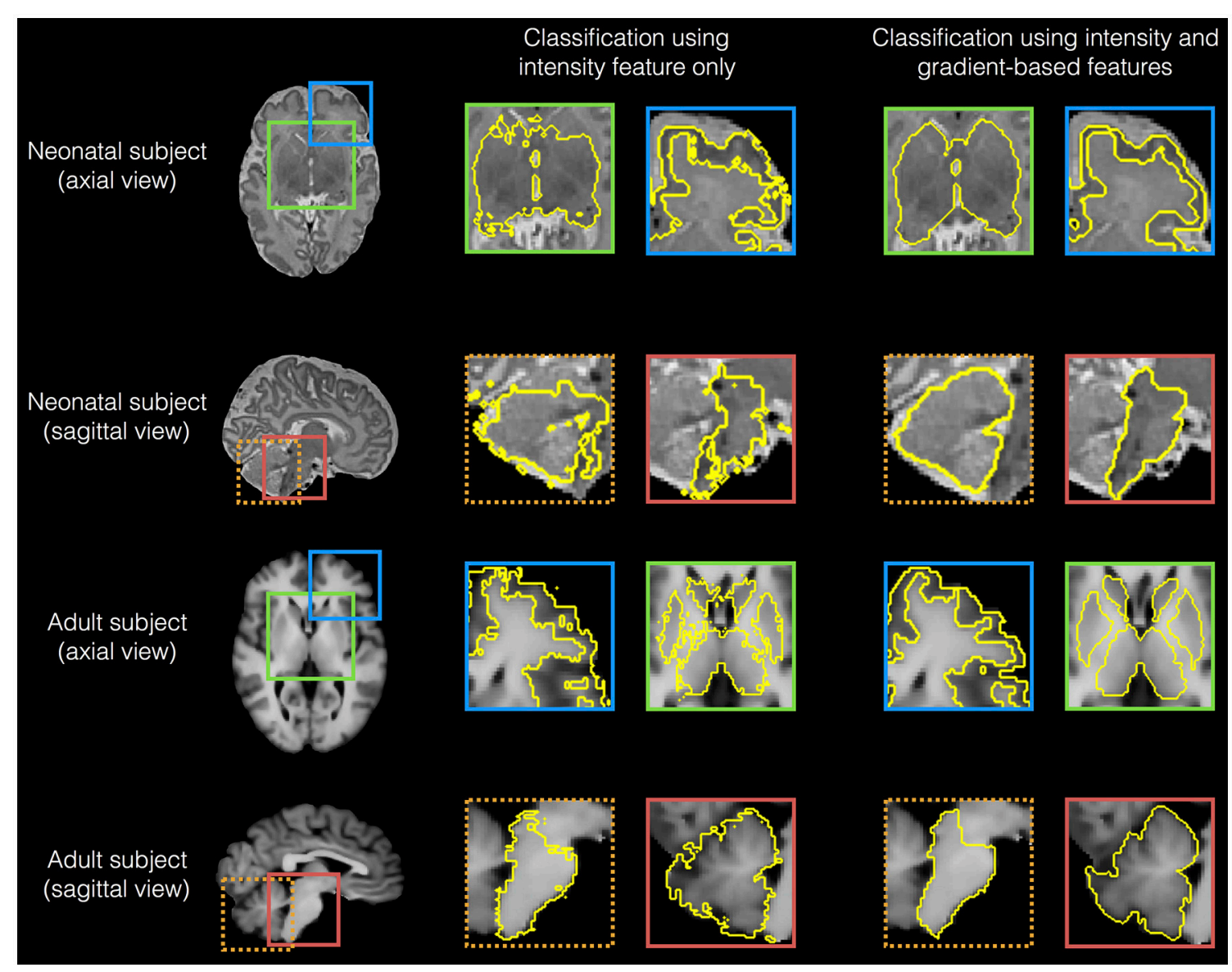

FIGURE 7 | Examples of edge detection for various regions (cortical gray matter, sub-cortical structures, brainstem and cerebellum) based on using all features (intensity combined with gradients) and intensity gray scale only, for a neonatal (dataset I) and an adult brain (dataset III).

\section{AUTHOR CONTRIBUTIONS}

AS designed and performed the experiments, and wrote the manuscript; AS, JB, and AGW analyzed output data; ET, RP, and SAS recruited patients; GM and SIS acquired imaging data. All authors approved the final submitted version, and agreed to be accountable for its content.

\section{FUNDING}

This work was supported by the Theirworld (http://www. theirworld.org), NHS Research Scotland, and NHS Lothian

\section{REFERENCES}

Aljabar, P., Heckemann, R. A., Hammers, A., Hajnal, J. V., and Rueckert, D. (2009). Multi-atlas based segmentation of brain images: atlas selection and its effect on accuracy. Neuroimage 46, 726-738. doi: 10.1016/j.neuroimage.2009.02.018

Altaye, M., Holland, S. K., Wilke, M., and Gaser, C. (2008). Infant brain probability templates for MRI segmentation and normalization. Neuroimage 43, 721-730. doi: 10.1016/j.neuroimage.2008.07.060
Research and Development. This work was undertaken in the MRC Centre for Reproductive Health which is funded by the MRC Centre grant MR/N022556/1.

\section{ACKNOWLEDGMENTS}

We are grateful to the families who consented to take part in the study and to the nursing and radiography staff at the Clinical Research Imaging Centre, University of Edinburgh (http://www.cric.ed.ac.uk) who participated in scanning the infants. 
Comput. Methods Programs Biomed. 104, e158-e177. doi: 10.1016/j.cmpb.2011. 07.015

Cai, W. L., Chen, S. C., and Zhang, D. Q. (2007). Fast and robust fuzzy c-means clustering algorithms incorporating local information for image segmentation. Pattern Recogn. 40, 825-838. doi: 10.1016/j.patcog.2006.07.011

Cardoso, M. J., Melbourne, A., Kendall, G. S., Modat, M., Robertson, N. J., Marlow, N., et al. (2013). AdaPT: an adaptive preterm segmentation algorithm for neonatal brain MRI. Neuroimage 65, 97-108. doi: 10.1016/j.neuroimage.2012.08.009

Cherel, M., Budin, F., Prastawa, M., Gerig, G., Lee, K., Buss, C., et al. (2015). Automatic tissue segmentation of neonate brain MR images with subjectspecific Atlases. Proc. SPIE Int. Soc. Opt. Eng. 9413. doi: 10.1117/12.2082209

Coupé, P., Manjón, J. V., Fonov, V., Pruessner, J., Robles, M., and Collins, D. L. (2011). Patch-based segmentation using expert priors: application to hippocampus and ventricle segmentation. Neuroimage 54, 940-954. doi: 10.1016/j.neuroimage.2010.09.018

Criminisi, A., and Shotton, J. (2013). Decision Forests for Computer Vision and Medical Image Analysis. London; New York, NY: Springer.

Despotovic, I., Goossens, B., and Philips, W. (2015). MRI segmentation of the human brain: challenges, methods, and applications. Comput. Math. Methods Med. 2015:450341. doi: 10.1155/2015/450341

Dice, L. R. (1945). Measures of the amount of ecologic association between species. Ecology 26, 297-302. doi: 10.2307/1932409

Fischl, B., Salat, D. H., Busa, E., Albert, M., Dieterich, M., Haselgrove, C., et al. (2002). Whole brain segmentation: automated labeling of neuroanatomical structures in the human brain. Neuron 33, 341-355. doi: 10.1016/S0896-6273(02)00569-X

Frazier, J. A., Hodge, S. M., Breeze, J. L., Giuliano, A. J., Terry, J. E., Moore, C. M., et al. (2008). Diagnostic and sex effects on limbic volumes in early-onset bipolar disorder and schizophrenia. Schizophr. Bull. 34, 37-46. doi: $10.1093 / \mathrm{schbul} / \mathrm{sbm} 120$

Geremia, E., Clatz, O., Menze, B. H., Konukoglu, E., Criminisi, A., and Ayache, N. (2011). Spatial decision forests for MS lesion segmentation in multi-channel magnetic resonance images. Neuroimage 57, 378-390. doi: 10.1016/j.neuroimage.2011.03.080

Gui, L., Lisowski, R., Faundez, T., Hüppi, P. S., Lazeyras, F., and Kocher, M. (2012). Morphology-driven automatic segmentation of MR images of the neonatal brain. Med. Image Anal. 16, 1565-1579. doi: 10.1016/j.media.2012.07.006

Heckemann, R. A., Hajnal, J. V., Aljabar, P., Rueckert, D., and Hammers, A. (2006). Automatic anatomical brain MRI segmentation combining label propagation and decision fusion. Neuroimage 33, 115-126. doi: 10.1016/j.neuroimage.2006.05.061

Hill, J., Dierker, D., Neil, J., Inder, T., Knutsen, A., Harwell, J., et al. (2010). A surface-based analysis of hemispheric asymmetries and folding of cerebral cortex in term-born human infants. J. Neurosci. 30, 2268-2276. doi: 10.1523/JNEUROSCI.4682-09.2010

Ho, T. K. (1998). The random subspace method for constructing decision forests. IEEE Trans. Pattern Anal. Mach. Intell. 20, 832-844. doi: 10.1109/34.709601

Huang, C., Ding, X., and Fang, C. (2010). "Head pose estimation based on random forests for multiclass classification," in 20th International Conference on Pattern Recognition (ICPR) (Istanbul), 934-937.

Iglesias, J. E., Liu, C. Y., Thompson, P. M., and Tu, Z. (2011). Robust brain extraction across datasets and comparison with publicly available methods. IEEE Trans. Med. Imaging 30, 1617-1634. doi: 10.1109/TMI.2011.2138152

Iglesias, J. E., and Sabuncu, M. R. (2015). Multi-atlas segmentation of biomedical images: a survey. Med. Image Anal. 24, 205-219. doi: 10.1016/j.media.2015.06.012

Išgum, I., Benders, M. J., Avants, B., Cardoso, M. J., Counsell, S. J., Gomez, E. F., et al. (2015). Evaluation of automatic neonatal brain segmentation algorithms: the NeoBrainS12 challenge. Med. Image Anal. 20, 135-151. doi: 10.1016/j.media.2014.11.001

Job, D. E., Dickie, D. A., Rodriguez, D., Robson, A., Danso, S., Pernet, C., et al. (2017). A brain imaging repository of normal structural MRI across the life course: Brain Images of Normal Subjects (BRAINS). Neuroimage 144, 299-304. doi: 10.1016/j.neuroimage.2016.01.027

Kaba, D., Wang, C., Li, Y., Salazar-Gonzalez, A., Liu, X. and Serag, A. (2014). Retinal blood vessels extraction using probabilistic modelling. Health Inf. Sci. Syst. 2:2. doi: 10.1186/2047-2501-2-2
Kennedy, D. N., Haselgrove, C., Hodge, S. M., Rane, P. S., Makris, N., and Frazier, J. A. (2012). CANDIShare: a resource for pediatric neuroimaging data. Neuroinform 10, 319-322. doi: 10.1007/s12021-011-9133-y

Kuklisova-Murgasova, M., Aljabar, P., Srinivasan, L., Counsell, S. J., Doria, V., Serag, A., et al. (2011). A dynamic 4D probabilistic atlas of the developing brain. Neuroimage 54, 2750-2763. doi: 10.1016/j.neuroimage.2010.10.019

Leroy, F., Mangin, J. F., Rousseau, F., Glasel, H., Hertz-Pannier, L., Dubois, J., et al. (2011). Atlas-free surface reconstruction of the cortical grey-white interface in infants. PLoS ONE 6:e27128. doi: 10.1371/journal.pone.0027128

Loh, W. Y., Connelly, A., Cheong, J. L., Spittle, A. J., Chen, J., Adamson, C., et al. (2016). A new MRI-based pediatric subcortical segmentation technique (PSST). Neuroinformatics 14, 69-81. doi: 10.1007/s12021-015-9279-0

Lötjönen, J. M., Wolz, R., Koikkalainen, J. R., Thurfjell, L., Waldemar, G., Soininen, H., et al. (2010). Fast and robust multi-atlas segmentation of brain magnetic resonance images. Neuroimage 49, 2352-2365. doi: 10.1016/j.neuroimage.2009.10.026

Makropoulos, A., Ledig, C., Aljabar, P., Serag, A., Hajnal, J. V., Edwards, A. D., et al. (2012). Automatic tissue and structural segmentation of neonatal brain MRI using expectation-maximization. MICCAI Grand Chall. Neonatal Brain Segmentation 2012, 9-15. Available online at: http://neobrains12.isi.uu.nl/pdf/ Imperial.pdf

Mazziotta, J., Toga, A., Evans, A., Fox, P., Lancaster, J., Zilles, K., et al. (2001). A probabilistic atlas and reference system for the human brain: International Consortium for Brain Mapping (ICBM). Philos. Trans. R. Soc. Lond. B. Biol. Sci. 356, 1293-1322. doi: 10.1098/rstb.2001.0915

McGurn, B., Deary, I. J., and Starr, J. M. (2008). Childhood cognitive ability and risk of late-onset Alzheimer and vascular dementia. Neurology 71, 1051-1056. doi: 10.1212/01.wnl.0000319692.20283.10

Mitra, J., Bourgeat, P., Fripp, J., Ghose, S., Rose, S., Salvado, O., et al. (2014). Lesion segmentation from multimodal MRI using random forest following ischemic stroke. Neuroimage 98, 324-335. doi: 10.1016/j.neuroimage.2014. 04.056

Modat, M., Ridgway, G. R., Taylor, Z. A., Lehmann, M., Barnes, J., Hawkes, D. J., et al. (2010). Fast free-form deformation using graphics processing units. Comput. Methods Programs Biomed. 98, 278-284. doi: $10.1016 /$ j.cmpb.2009.09.002

Moeskops, P., Benders, M. J., Chit, S. M., Kersbergen, K. J., Groenendaal, F., de Vries, L. S., et al. (2015). Automatic segmentation of MR brain images of preterm infants using supervised classification. Neuroimage 118, 628-641. doi: 10.1016/j.neuroimage.2015.06.007

Nyul, L. G., and Udupa, J. K. (2000). Standardizing the MR image intensity scales: making MR intensities have tissue-specific meaning. Proc. SPIE Int. Soc. Opt. Eng. 3976, 496-504. doi: 10.1117/12.383076

Pereira, S., Pinto, A., Oliveira, J., Mendrik, A. M., Correia, J. H., and Silva, C. A. (2016). Automatic brain tissue segmentation in MR images using random forests and conditional random fields. J. Neurosci. Meth. 270, 111-123. doi: 10.1016/j.jneumeth.2016.06.017

Prastawa, M., Gilmore, J. H., Lin, W., and Gerig, G. (2005). Automatic segmentation of MR images of the developing newborn brain. Med. Image Anal. 9, 457-466. doi: 10.1016/j.media.2005.05.007

Rohlfing, T. (2012). Image similarity and tissue overlaps as surrogates for image registration accuracy: widely used but unreliable. IEEE Trans. Med. Imaging 31, 153-163. doi: 10.1109/TMI.2011.2163944

Rohlfing, T., Brandt, R., Menzel, R., and Maurer, C. R. Jr. (2004). Evaluation of atlas selection strategies for atlas-based image segmentation with application to confocal microscopy images of bee brains. Neuroimage 21, 1428-1442. doi: 10.1016/j.neuroimage.2003.11.010

Rousseau, F., Habas, P. A., and Studholme, C. (2011). A supervised patch-based approach for human brain labeling. IEEE Trans. Med. Imaging 30, 1852-1862. doi: 10.1109/TMI.2011.2156806

Rueckert, D., Sonoda, L. I., Hayes, C., Hill, D. L. G., Leach, M. O., and Hawkes, D. J. (1999). Nonrigid registration using free-form deformations: application to breast MR images. IEEE Trans. Med. Imaging 18, 712-721. doi: 10.1109/42.796284

Serag, A., Aljabar, P., Ball, G., Counsell, S. J., Boardman, J. P., Rutherford, M. A., et al. (2012a). Construction of a consistent high-definition spatio-temporal atlas of the developing brain using adaptive kernel regression. Neuroimage 59, 2255-2265. doi: 10.1016/j.neuroimage.2011.09.062 
Serag, A., Blesa, M., Moore, E. J., Pataky, R., Sparrow, S., Wilkinson, A. G., et al. (2016). Accurate Learning with Few Atlases (ALFA): an algorithm for MRI neonatal brain extraction and comparison with 11 publicly available methods. Sci. Rep. 6:23470. doi: 10.1038/srep23470

Serag, A., Gousias, I. S., Makropoulos, A., Aljabar, P., Hajnal, J. V., Boardman, J. P., et al. (2012b). "Unsupervised learning of shape complexity: application to brain development," in MICCAI Workshop on Spatio-Temporal Image Analysis for Longitudinal and Time-Series Image Data (Nice).

Serag, A., Kyriakopoulou, V., Rutherford, M. A., Edwards, A. D., Hajnal, J. V., Aljabar, P., et al. (2012c). A multi-channel 4D probabilistic Atlas of the developing brain: application to fetuses and neonates. Ann. BMVA 2012, 1-14. Avilable online at: http://www.bmva.org/annals/2012/2012-0003.pdf

Shenkin, S. D., Bastin, M. E., Macgillivray, T. J., Deary, I. J., Starr, J. M., and Wardlaw, J. M. (2009). Birth parameters are associated with late-life white matter integrity in community-dwelling older people. Stroke 40, 1225-1228. doi: 10.1161/STROKEAHA.108.527259

Shi, F., Yap, P. T., Fan, Y., Gilmore, J. H., Lin, W., and Shen, D. (2010). Construction of multi-region-multi-reference atlases for neonatal brain MRI segmentation. Neuroimage 51, 684-693. doi: 10.1016/j.neuroimage.2010.02.025

Song, Z., Awate, S. P., Licht, D. J., and Gee, J. C. (2007). Clinical neonatal brain MRI segmentation using adaptive nonparametric data models and intensity-based Markov priors. Med. Image Comput. Comput. Assist. Interven. 4791, 883-890. doi: 10.1007/978-3-540-75757-3_107

Stoner, R., Chow, M. L., Boyle, M. P., Sunkin, S. M., Mouton, P. R., Roy, S., et al. (2014). Patches of disorganization in the neocortex of children with autism. $N$. Engl. J. Med. 370, 1209-1219. doi: 10.1056/NEJMoa1307491

Tamnes, C. K., Walhovd, K. B., Dale, A. M., Østby, Y., Grydeland, H., Richardson, G., et al. (2013). Brain development and aging: overlapping and unique patterns of change. Neuroimage 68, 63-74. doi: 10.1016/j.neuroimage.2012.11.039

Tustison, N. J., Avants, B. B., Cook, P. A., Yuanjie, Z., Egan, A., Yushkevich, P. A., et al. (2010). N4ITK: improved N3 bias correction. IEEE Trans. Med. Imaging 29, 1310-1320. doi: 10.1109/TMI.2010.2046908

Tustison, N. J., Shrinidhi, K. L., Wintermark, M., Durst, C. R., Kandel, B. M., Gee, J. C., et al. (2015). Optimal symmetric multimodal templates and concatenated random forests for supervised brain tumor segmentation (Simplified) with ANTsR. Neuroinform 13, 209-225. doi: 10.1007/s12021-014-9245-2

Van Leemput, K., Maes, F., Vandermeulen, D., Colchester, A., and Suetens, P. (2001). Automated segmentation of multiple sclerosis lesions by model outlier detection. IEEE Trans. Med. Imaging 20, 677-688. doi: 10.1109/42.938237

Vovk, A., Cox, R. W., Stare, J., Suput, D., and Saad, Z. S. (2011). Segmentation priors from local image properties: without using bias field correction, location-based templates, or registration. Neuroimage 55, 142-152. doi: 10.1016/j.neuroimage.2010.11.082
Wang, L., Gao, Y., Shi, F., Li, G., Gilmore, J. H., Lin, W., et al. (2015). LINKS: Learning-based multi-source IntegratioN frameworK for Segmentation of infant brain images. Neuroimage 108, 160-172. doi: 10.1016/j.neuroimage.2014.12.042

Wardlaw, J. M., Bastin, M. E., Valdés Hernández, M. C., Maniega, S. M., Royle, N. A., Morris, Z., et al. (2011). Brain aging, cognition in youth and old age and vascular disease in the Lothian Birth Cohort 1936: rationale, design and methodology of the imaging protocol. Int. J. Stroke 6, 547-559. doi: 10.1111/j.1747-4949.2011.00683.x

Warfield, S. K., Zou, K. H., and Wells, W. M. (2004). Simultaneous truth and performance level estimation (STAPLE): an algorithm for the validation of image segmentation. IEEE Trans. Med. Imaging 23, 903-921. doi: 10.1109/TMI.2004.828354

Weglinski, T., and Fabijanska, A. (2011). "Brain tumor segmentation from MRI data sets using region growing approach," in Proceedings of VIIth International Conference on Perspective Technologies and Methods in MEMS Design (MEMSTECH) (Polyana), 185-188.

Weisenfeld, N. I., and Warfield, S. K. (2009). Automatic segmentation of newborn brain MRI. Neuroimage 47, 564-572. doi: 10.1016/j.neuroimage.2009. 04.068

Weiss, G. M., and Provost, F. (2003). Learning when training data are costly: the effect of class distribution on tree induction. J. Artif. Intell. Res. 19, 315-354. Available online at: https://www.jair.org/media/1199/live-11992209-jair.pdf

Yi, Z., Criminisi, A., Shotton, J., and Blake, A. (2009). Discriminative, semantic segmentation of brain tissue in MR Images. Med. Image Comput. Comput. Assist. Interven. 5762, 558-565. doi: 10.1007/978-3-64204271-3_68

Zikic, D., Glocker, B., and Criminisi, A. (2014). Encoding atlases by randomized classification forests for efficient multi-atlas label propagation. Med. Image Anal. 18, 1262-1273. doi: 10.1016/j.media.2014.06.010

Conflict of Interest Statement: The authors declare that the research was conducted in the absence of any commercial or financial relationships that could be construed as a potential conflict of interest.

Copyright (c) 2017 Serag, Wilkinson, Telford, Pataky, Sparrow, Anblagan, Macnaught, Semple and Boardman. This is an open-access article distributed under the terms of the Creative Commons Attribution License (CC BY). The use, distribution or reproduction in other forums is permitted, provided the original author(s) or licensor are credited and that the original publication in this journal is cited, in accordance with accepted academic practice. No use, distribution or reproduction is permitted which does not comply with these terms. 